\title{
Important role of three-body repulsive force effect in nuclear reactions
}

\author{
T. Furumoto ${ }^{1,2, a}$, Y. Sakuragi ${ }^{1,2, b}$, and Y. Yamamoto ${ }^{3, c}$ \\ 1 Department of Physics, Osaka City University, Osaka 558-8585, Japan \\ 2 RIKEN Nishina Center, RIKEN, Wako, Saitama 351-0198, Japan \\ 3 Physics Section, Tsuru University, Tsuru, Yamanashi 402-8555, Japan
}

\begin{abstract}
The effect of three-body force (TBF) is studied in nucleus-nucleus elastic scattering on the basis of Brueckner theory for nucleon-nucleon $(N N)$ effective interaction (complex $G$ matrix) in the nuclear matter. A new $G$ matrix called CEG07 proposed recently by the present authors includes the TBF effect and reproduces a realistic saturation curve in the nuclear matter, and is shown to well reproduce proton-nucleus elastic scattering. The microscopic optical potential for nucleus-nucleus system is obtained by folding the $G$ matrix with nucleon density distributions in colliding nuclei. We first analyze the ${ }^{16} \mathrm{O}+{ }^{16} \mathrm{O}$ elastic scattering at $E / A=70 \mathrm{MeV}$ in detail. The observed cross sections are nicely reproduced up to the most backward scattering angles only when the TBF effect is included. The effects of the three-body attraction (TBA) and three-body repulsion (TBR) are also analyzed. The TBR contribution has an important role in nucleus-nucleus elastic scattering. The CEG07 $G$ matrix is also tested in the elastic scattering of ${ }^{16} \mathrm{O}$ by the ${ }^{12} \mathrm{C},{ }^{28} \mathrm{Si}$ and ${ }^{40} \mathrm{Ca}$ targets at $E / A=93.9 \mathrm{MeV}$, and in the elastic scattering of ${ }^{12} \mathrm{C}$ by the ${ }^{12} \mathrm{C}$ target at $E / A=135 \mathrm{MeV}$ with a great success. The decisive effect of the TBF is clearly seen also in those systems.
\end{abstract}

\section{Introduction}

The role of the nuclear three-body force (TBF) in complex nuclear systems is one of the key issues not only in nuclear physics but also in nuclear astrophysics relevant to high-density nuclear matter in neutron stars and supernova explosions. It is well known that the empirical saturation point of nuclear matter (the binding energy per nucleon $E / A \approx 16 \mathrm{MeV}$ at a saturation density $\rho_{0} \approx 0.17 \mathrm{fm}^{-3}$ ) cannot be reproduced by using only two-body nucleonnucleon $(N N)$ interactions [1]. To obtain a reasonable saturation curve, it is indispensable to take into account the additional contributions of the TBF, which contains two parts: a three-body attraction (TBA) and a three-body repulsion (TBR). It is important here that the saturation curve in the high-density region is strongly pushed upward by the TBR contribution and, as the result, the nuclear-matter incompressibility becomes large [2-5]. This effect is intimately related to our problem. In Ref. [6], we reported for the first time clear evidence of the important role of the TBF (especially the TBR) in nucleus-nucleus elastic scattering in the case of an ${ }^{16} \mathrm{O}+{ }^{16} \mathrm{O}$ system at $E / A=70 \mathrm{MeV}$.

Understanding nucleon-nucleus $(N A)$ and nucleus-nucleus $(A A)$ interactions microscopically starting from underlying $N N$ interactions has been a longstanding and fundamental subject. To solve a complicated many-body problem in nuclear reactions, one needs to rely upon a realistic approach based on reasonable approximations. One

\footnotetext{
a e-mail: furumoto@ocunp.hep.osaka-cu.ac.jp

b e-mail: sakuragi@ocunp.hep.osaka-cu.ac.jp

c e-mail: yamamoto@tsuru.ac.jp
}

of the promising approaches would be to derive the $N A$ and $A A$ folding potentials on the basis of the lowest order Brueckner theory. Here, the $N N G$-matrix interactions are obtained in infinite nuclear matter and folded into $N A$ and $A A$ density distributions with the local-density approximation (LDA). The Bethe-Goldstone (B-G) equation is solved for an $N N$ pair in medium, one of which corresponds to an incident nucleon and the other is under a scattering boundary condition. The obtained $G$-matrix interaction is composed of real and imaginary parts, being dependent on the incident energy and the nuclear-matter density. As noted here, the $G$ matrix is considered to be an effective $N N$ interaction in nuclear medium, into which the short-range and tensor correlations are renormalized.

The folding-model study with the use of complex $G$ matrix interactions for $N A$ system has a long history. Various $G$-matrix interactions starting from different kinds of free-space $N N$ interactions were proposed and applied to the analyses of proton-nucleus elastic scattering with more or less successful results. However, all of the $G$ matrix proposed so far were derived from just the two-body force and the effect of the TBF was not included nor discussed. This is partly because the TBF contributions at densities lower than $\rho_{0}$ have been considered to be not large enough to affect $N A$ scattering observables. Here, one should note that the local density felt by the incident nucleon inside the target nucleus does not exceed $\rho_{0}$ even deep inside the nucleus in usual cases.

Recently, the present authors have proposed a new complex $G$-matrix interaction $C E G 07$ [7] derived from the extended soft-core (ESC) model $[9,10]$. The ESC model is 
designed to give a consistent description of interactions not only for the $N N$ system but also for nucleon-hyperon and hyperon-hyperon systems. In this model, the TBR effect is represented as density-dependences of two-body parts which appear by changing vector-meson masses in a densitydependent way [10]. In contrast, the TBA part typically results from two-pion exchange with excitation of an intermediate $\Delta$ resonance, that is, the Fujita-Miyazawa diagram [11], which gives an important contribution at low densities. Although the saturation curve of nuclear matter can be produced reasonably as combined contributions of the TBA and the TBR, it is decisively important in our results that the TBR contribution becomes more and more significant as the density increases. The CEG07 models were first applied to the analysis of proton-nucleus elastic scattering over a wide range of incident energies and target nuclei with great success [7]. Although the inclusion of the TBF effect, in general, gave rise to only minor change of $p A$ elastic-scattering cross sections, as expected from our earlier discussion, it was demonstrated that the inclusion of the TBF effect clearly improved the fit to the analyzing power data at forward angles in some energy regions.

In the case of the $A A$ scattering system, the local density $\left(\rho_{1}+\rho_{2}\right)$ in the projectile-target overlap region may exceed the normal density of nuclear matter, $\rho_{0}$, and could reach about twice this value, under the frozen-density approximation (FDA), as mentioned later. The TBR contributions are remarkably large in such high-density regions and, hence, one may expect clear evidence of a TBR effect through the calculated folding-model potential (FMP) and the resultant elastic-scattering observables. The importance of a consistent description of nuclear saturation properties and elastic scattering of $A A$ systems was first pointed out by Khoa et al. $[12,13]$ on the basis of folding-model analyses. They used density-dependent effective $N N$ interactions such as DDM3Y, BDM3Y, and CDM3Y obtained from the density-independent effective interaction M3Y [14] by multiplying various kind of phenomenological density-dependent factors by hand, the parameters of which were chosen so as to represent various types of saturation curves in nuclear matter. The real part of the $A A$ potential was calculated by the folding of these interactions with nucleon densities of the $A A$ system, whereas the imaginary part was treated in a completely phenomenological way because M3Y was composed only of a real part. They showed the importance of using an effective interaction to be chosen to reproduce the realistic saturation curve in nuclear matter for the proper description of elastic scattering of $A A$ systems. However, their purely phenomenological density-dependent factor had no explicit nor logical relation to the TBF in the nuclear medium.

The present paper is organized as follows: Sec. 2 gives the expressions of the $G$-matrix interaction and the doublefolding model for a nucleus-nucleus potential with the use of the complex $G$-matrix interactions. Our analyses for various scattering $A A$ systems are given in Sec. 3. Here, the importance of the TBF effect (especially the TBR) is demonstrated in the typical case of the ${ }^{16} \mathrm{O}+{ }^{16} \mathrm{O}$ elastic scattering at $E / A=70 \mathrm{MeV}$, and then the analyses are performed for the elastic scattering of ${ }^{16} \mathrm{O}$ on the ${ }^{12} \mathrm{C},{ }^{28} \mathrm{Si}$, and ${ }^{40} \mathrm{Ca}$ targets at $E / A=93.9 \mathrm{MeV}$, for the ${ }^{12} \mathrm{C}+{ }^{12} \mathrm{C}$ elastic scattering at $E / A=135 \mathrm{MeV}$. The summary of this work is given in Sec. 4.

\section{Formalism}

\subsection{G-matrix interaction}

Let us recapture the derivation of the $G$-matrix interaction given in our previous work $[7,8]$. We start from the $G$ matrix equation for the nucleon pair of the moving nucleon with momentum, $\boldsymbol{k}$, and a bound nucleon with momentum, $\boldsymbol{k}_{\boldsymbol{j}}$, in symmetric nuclear matter at the Fermi-momentum, $k_{\mathrm{F}}$, where the starting energy, $\omega$, is given as the sum of the energy $E(k)$ of the propagating nucleon and a single particle (s.p.) energy $e\left(k_{j}\right)$. The $G$-matrix calculations are performed with the continuous choice for intermediate nucleon spectra. The scattering boundary condition with $i \varepsilon$ in the denominator leads to complex $G$ matrices, summation of which gives the complex s.p. potential $U(k, E(k))$. Then, the energy $E(k)=\frac{\hbar^{2}}{2 m} k^{2}+U_{R}(k)$ is determined selfconsistently, $U_{R}(k)$ being the real part of $U(k, E(k))$.

Relative and center-of-mass momenta are given as $\boldsymbol{q}=$ $\left(\boldsymbol{k}-\boldsymbol{k}_{\boldsymbol{j}}\right) / 2$ and $\boldsymbol{P}=\boldsymbol{k}+\boldsymbol{k}_{\boldsymbol{j}}$, respectively. Angular momenta of relative-orbital, spin and total states are denoted by $L, S$ and $J$, respectively, and isospin is done by $T$. Then, the coordinate-space $G$-matrix equation in a $(L, S, J, T)$ pair state is represented as follows:

$$
\begin{aligned}
& u_{L L^{\prime}}^{J S T}(r ; q)=j_{L}(q r) \delta_{L L^{\prime}} \\
& +4 \pi \sum_{L^{\prime \prime}} \int r^{\prime 2} d r^{\prime} F_{L}\left(r, r^{\prime} ; q\right) V_{L^{\prime} L^{\prime \prime}}^{J S T}\left(r^{\prime}\right) u_{L L^{\prime \prime}}^{J S T}\left(r^{\prime} ; q\right), \\
& F_{L}\left(r, r^{\prime} ; q\right)=\frac{1}{2 \pi^{2}} \int q^{\prime 2} d q^{\prime} \\
& \times \frac{\bar{Q}\left(q^{\prime}, \bar{P} ; k_{F}\right) j_{L}\left(q^{\prime} r\right) j_{L}\left(q^{\prime} r^{\prime}\right)}{\omega-\left(\frac{\hbar^{2}}{m} q^{\prime 2}+\frac{\hbar^{2}}{4 m} \bar{P}^{2}+U_{R}\left(\bar{q}_{+}^{\prime}\right)+U_{R}\left(\bar{q}_{-}^{\prime}\right)\right)},
\end{aligned}
$$

where $j_{L}(q r)$ is a spherical Bessel function for the incident momentum, $q$, and $u_{L L^{\prime}}^{J S T}(r ; q)$ is the corresponding scattering wave function. $\bar{Q}, \bar{P}$ and $\bar{q}_{ \pm}^{\prime}$ are the angle-averaged expressions for the Pauli operator, $Q$, the center-of-mass momentum, $P$, and $\left|\boldsymbol{q}^{\prime} \pm \frac{1}{2} \boldsymbol{P}\right|$, respectively. The $G$-matrix elements and the s.p. potentials are given as

$$
\begin{aligned}
& \left\langle q\left|G_{L L}^{J S T}\right| q\right\rangle=4 \pi \sum_{L^{\prime \prime}} \int r^{2} d r j_{L}(q r) V_{L L^{\prime \prime}}^{J S T} u_{L L^{\prime \prime}}^{J S T}(r ; q), \\
& U\left(k, E(k) ; k_{F}\right)=\frac{1}{2 \pi^{2}} \int q^{2} d q Z\left(q ; k, k_{F}\right) \\
& \times \sum_{L J S T} \frac{1}{2}(2 J+1)(2 T+1)\left\langle q\left|G_{L L}^{J S T}\right| q\right\rangle,
\end{aligned}
$$

with

$$
Z\left(q ; k, k_{F}\right)=\frac{1}{k q}\left(k_{F}^{2}-(k-2 q)^{2}\right) .
$$


The $G$-matrix interaction represented as a local form in the coordinate space can be given as follows:

$$
\begin{aligned}
& G_{L L^{\prime}}^{J S T}\left(r ; k_{F}, E\right) \\
& =\frac{\int q^{2} d q Z\left(q ; k, k_{F}\right) j_{L^{\prime}}(q r) \sum_{L^{\prime \prime}} V_{L^{\prime} L^{\prime \prime}}^{J S T}(r) u_{L L^{\prime \prime}}^{J S T}(r ; q)}{\int q^{2} d q Z\left(q ; k, k_{F}\right) j_{L^{\prime}}(q r) j_{L}(q r)}
\end{aligned}
$$

where the $q$-dependence in the $G$-matrix (2) is averaged over so as to reproduce the s.p. potential (3) in the firstorder perturbation. The apparent $k$-dependence in the right side is attributed to the $E$-dependence through the relation $E(k)=\frac{\hbar^{2}}{2 m} k^{2}+U_{R}(k)$. The obtained interaction $G_{L L}^{J S T}(r)$ is parameterized in a three-range Gaussian form: The outer two-ranges are determined by fitting the radial form of $G_{L L}^{J S T}(r)$ in long- and intermediate-range regions, and the innermost part is fixed so as to reproduce the ( $L S J T)$-state contribution of the s.p. potential $U$. The central and $L S$ components of the $G$-matrix interaction in the $(L S T)$ state are given by the adequate linear combinations of $G_{L L}^{J S T}$ on $J$. The $L$-dependence is further averaged for each parity state with the statistical weight given by the denominator of Eq. (5). Thus, we obtain the three-Gaussian potential $G_{ \pm}^{S T}(r), \pm$ being even and odd parities. Our Gaussianparameterized $G$-matrix interaction is named CEG07.

As for the $N N$ interaction model, we adopt the extended soft core (ESC) model $[9,10]$. Though many $N N$ interaction models have been proposed so far, the recent models reproduce the experimental phase shifts equally well. The $G$-matrix interactions derived from these models are considered to give rise to similar results for the nucleon-nucleus scattering observables. A reason for adopting ESC here is in the nuclear saturation problem. As well known, the empirical saturation point can be reproduced with the use of not only the two-body $N N$ interactions but also the three-body force (TBF) composed of the threebody attraction (TBA) and the three-body repulsion (TBR). The TBA is typically due to the two-pion exchange with excitation of an intermediate $\Delta$ resonance, that is the FujitaMiyazawa diagram. We derive the effective two-body interaction from the TBA, which is added on our $G$-matrix interaction, according to the formalism in Ref. [15]. In our calculations, the pionic form-factor mass is taken as 420 $\mathrm{MeV}$ and the $N N$ correlation effect for the TBA is neglected. On the other hand, the origin of the TBR is not necessarily established. In the ESC approach, the TBR effect is included rather phenomenologically by changing the vector-meson masses, $M_{V}$, in the nuclear matter according to $M_{V}(\rho)=M_{V} \exp \left(-\alpha_{V} \rho\right)$ with the parameter, $\alpha_{V}$, which leads to an effective density-dependent two-body interaction. In this work, we take the value of $\alpha_{V}=0.18$. Now, figure 1 shows the saturation curves in the following two cases:

(a) with the two-body interaction only,

(b) with TBA and TBR $\left(\alpha_{V}=0.18\right)$,

In the cases of (a) and (b), the minimum values of saturation curves are -17.8 and $-14.5 \mathrm{MeV}$, respectively, at $k_{F}=$ 1.53 and $1.33 \mathrm{fm}^{-1}$. Correspondingly, the calculated values of the incompressibility, $K$, at the normal density are obtained as 106 and $259 \mathrm{MeV}$, respectively. In the cases of

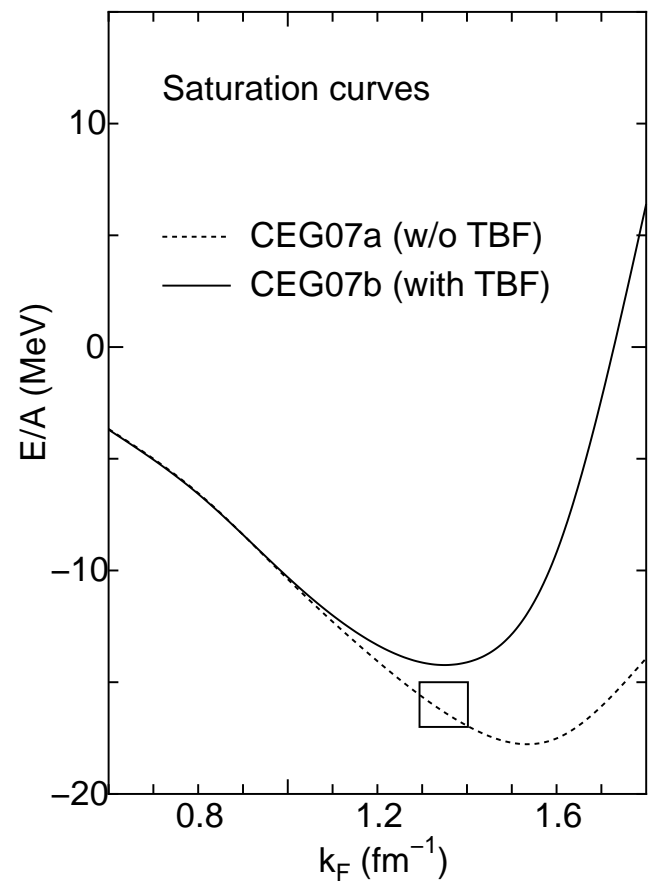

Fig. 1. Effects of the three-body forces in the saturation caurves obtained by the $G$-matrix calculation.

(a) and (b), the derived CEG interactions were named as CEG07a and CEG07b.

\subsection{Folding potential}

We construct the nucleus-nucleus optical model potential (OMP) based on the double-folding model (DFM) with the use of the complex $G$-matrix interaction CEG07. The microscopic nucleus-nucleus potential can be written as a Hartree-Fock type potential;

$$
\begin{aligned}
U_{\mathrm{F}} & =\sum_{i \in A_{1}, j \in A_{2}}\left[<i j\left|v_{\mathrm{D}}\right| i j>+<i j\left|v_{\mathrm{EX}}\right| j i>\right] \\
& =U_{\mathrm{D}}+U_{\mathrm{EX}},
\end{aligned}
$$

where $v_{\mathrm{D}}$ and $v_{\mathrm{EX}}$ are the direct and exchange parts of complex $G$-matrix interaction. The exchange part is a nonlocal potential in general. However, by the plane-wave representation for the $N N$ relative motion $[16,17]$, the exchange part can be localized. The direct and exchange parts of the localized potential are then written in the standard form of the DFM potential as

$$
U_{\mathrm{D}}(R)=\int \rho_{1}\left(\boldsymbol{r}_{1}\right) \rho_{2}\left(\boldsymbol{r}_{2}\right) v_{\mathrm{D}}(s ; \rho, E / A) d \boldsymbol{r}_{1} d \boldsymbol{r}_{2},
$$

where $\boldsymbol{s}=\boldsymbol{r}_{2}-\boldsymbol{r}_{1}+\boldsymbol{R}$, and

$$
\begin{aligned}
U_{\mathrm{EX}}(R)= & \int \rho_{1}\left(\boldsymbol{r}_{1}, \boldsymbol{r}_{1}+\boldsymbol{s}\right) \rho_{2}\left(\boldsymbol{r}_{2}, \boldsymbol{r}_{2}-\boldsymbol{s}\right) v_{\mathrm{EX}}(s ; \rho, E / A) \\
& \times \exp \left[\frac{i \boldsymbol{k}(R) \cdot \boldsymbol{s}}{M}\right] d \boldsymbol{r}_{1} d \boldsymbol{r}_{2} .
\end{aligned}
$$


Here, $\boldsymbol{k}(R)$ is the local momentum for nucleus-nucleus relative motion defined by

$$
k^{2}(R)=\frac{2 m M}{\hbar^{2}}\left[E_{\text {c.m. }}-\operatorname{Re} U_{\mathrm{F}}(R)-V_{\text {coul }}(R)\right],
$$

where $M=A_{1} A_{2} /\left(A_{1}+A_{2}\right), E_{\text {c.m. }}$ is the center-of-mass energy, $E / A$ is the incident energy per nucleon, $m$ is the nucleon mass and $V_{\text {coul }}$ is the Coulomb potential. $A_{1}$ and $A_{2}$ are the mass numbers of the projectile and target, respectively. The exchange part is calculated self-consistently on the basis of the local energy approximation (LDA) through Eq. (10). Here, the Coulomb potential $V_{\text {coul }}$ is also obtained by folding the $N N$ Coulomb potential with the proton density distributions of the projectile and target nuclei. The density matrix $\rho\left(\boldsymbol{r}, \boldsymbol{r}^{\prime}\right)$ is approximated in the same manner as in [18];

$$
\rho\left(\boldsymbol{r}, \boldsymbol{r}^{\prime}\right)=\frac{3}{k_{\mathrm{F}}^{\mathrm{eff}} \cdot s} j_{1}\left(k_{\mathrm{F}}^{\mathrm{eff}} \cdot s\right) \rho\left(\frac{\boldsymbol{r}+\boldsymbol{r}^{\prime}}{2}\right),
$$

where $k_{\mathrm{F}}^{\mathrm{eff}}$ is the effective Fermi momentum [19] defined by

$$
k_{\mathrm{F}}^{\mathrm{eff}}=\left(\left(3 \pi^{2} \rho\right)^{2 / 3}+\frac{5 C_{\mathrm{s}}\left[\nabla \rho^{2}\right]}{3 \rho^{2}}+\frac{5 \nabla^{2} \rho}{36 \rho}\right)^{1 / 2},
$$

where we adopt $C_{\mathrm{s}}=1 / 4$ following Ref. [20]. The detailed methods for calculating $U_{\mathrm{D}}$ (direct part) and $U_{\mathrm{EX}}$ (exchange part) are the same as those given in Refs. [21] and [12], respectively.

In the present calculations, we employ the so-called frozen density approximation (FDA) for evaluating the local density. In the FDA, the density-dependent $N N$ interaction is assumed to feel the local density defined as the sum of densities of colliding nuclei evaluated at the mid-point of the interacting nucleon pair;

$$
\rho=\rho_{1}\left(\boldsymbol{r}_{1}+\frac{1}{2} s\right)+\rho_{2}\left(\boldsymbol{r}_{2}-\frac{1}{2} s\right) .
$$

The FDA has been widely used also in the standard DFM calculations $[8,12,13,20,22,23]$. The prescriptions other than the FDA for evaluating the local density in $A A$ systems have already been tested in Ref. [8] and the prescription of the FDA has been confirmed to be the best approximation in the use of CEG07 interactions.

\section{Results}

Let us apply the CEG07 $G$-matrix interactions to nucleusnucleus $(A A)$ systems through the double-folding model (DFM). Since the imaginary part of the optical potential for $A A$ systems represents all excurrent flux escaping from elastic scattering channel through all the possible open reaction channels, it would be difficult to completely simulate those flux loss by the imaginary part of the $G$-matrix interaction originated from the pair-scattering correlations in the nuclear matter. So, we introduce a renormalization factor, $N_{\mathrm{W}}$, phenomenologically for the imaginary part of the FMP and define the present microscopic optical potential with the CEG07 interaction as

$$
U_{\mathrm{opt}}(R)=V(R)+i N_{\mathrm{W}} W(R) .
$$

Here, $V$ and $W$ denote the real and imaginary parts of the original DFM potential derived from the $G$-matrix interaction. We adjust the renormalization factor so as to attain optimum fits to the experimental data for elastic-scattering cross sections.

First, we analyze elastic scattering of the ${ }^{16} \mathrm{O}+{ }^{16} \mathrm{O}$ system in detail as a benchmark system for testing the interaction model, and then, we also analyze the ${ }^{16} \mathrm{O}$ scattering by other target nuclei as well as the ${ }^{12} \mathrm{C}+{ }^{12} \mathrm{C}$ system. We adopt the nucleon density of ${ }^{16} \mathrm{O}$ calculated from the internal wave functions generated by the orthogonal condition model (OCM) by Okabe [27] based on the microscopic $\alpha+{ }^{12} \mathrm{C}$ cluster picture. For other nuclei, we use the nucleon densities deduced from the charge densities [28] extracted from electron-scattering experiments by unfolding the charge form factor of a proton in the standard way [29].

\section{$3.1^{16} \mathrm{O}+{ }^{16} \mathrm{O}$ elastic scattering}

In this paper, we make use of the CEG07 $G$-matrix interactions with and without the TBF effect, and analyze the elastic scattering of the ${ }^{16} \mathrm{O}+{ }^{16} \mathrm{O}$ system to see how the TBF effect plays an important role in $A A$ scattering systems. We choose elastic scattering of the ${ }^{16} \mathrm{O}+{ }^{16} \mathrm{O}$ system for the following reasons: ${ }^{16} \mathrm{O}$ is one of the most stable double-magic nuclei and has no collective-excitation state strongly coupled with the ground state. This is important for a folding model based on the complex $G$-matrix, because the imaginary part of the $G$-matrix is expected to simulate the effect of single-particle-like excitations of finite nuclear systems through $N N$ pair-scattering correlations and the effect of coherent, collective excitations of a finite nucleus may not be included in the imaginary part of the $G$-matrix. Therefore, the ${ }^{16} \mathrm{O}+{ }^{16} \mathrm{O}$ system is one of the ideal benchmark systems to test the validity of interaction models; in fact, a number of interaction models, either purely phenomenological [30-32] or microscopic in various senses $[12,13,22,23]$, have been tested for decades on this system as a milestone to be cleared.

We now analyze the ${ }^{16} \mathrm{O}+{ }^{16} \mathrm{O}$ elastic scattering at $E / A$ $=70 \mathrm{MeV}$, paying a special attention to the roles of the TBF in $A A$ scattering systems. Figure 2 shows the real (upper panel) and imaginary (lower panel) parts of the calculated FMP for the ${ }^{16} \mathrm{O}+{ }^{16} \mathrm{O}$ elastic scattering at $E / A=$ $70 \mathrm{MeV}$ with the use of two types of CEG07 $G$-matrix interactions; CEG07a (without the TBF) and CEG07b (with the TBF). The effect of the TBF composed of the TBA and the TBR is clearly seen in the real part of the FMP over the whole range of internuclear distance, while the effect on the imaginary part is rather small except at short distances below $3 \mathrm{fm}$. The large difference in the real part is found to be mainly due to the effect of the repulsive part of TBF (TBR), while the attractive part of the TBF (TBA) is found 


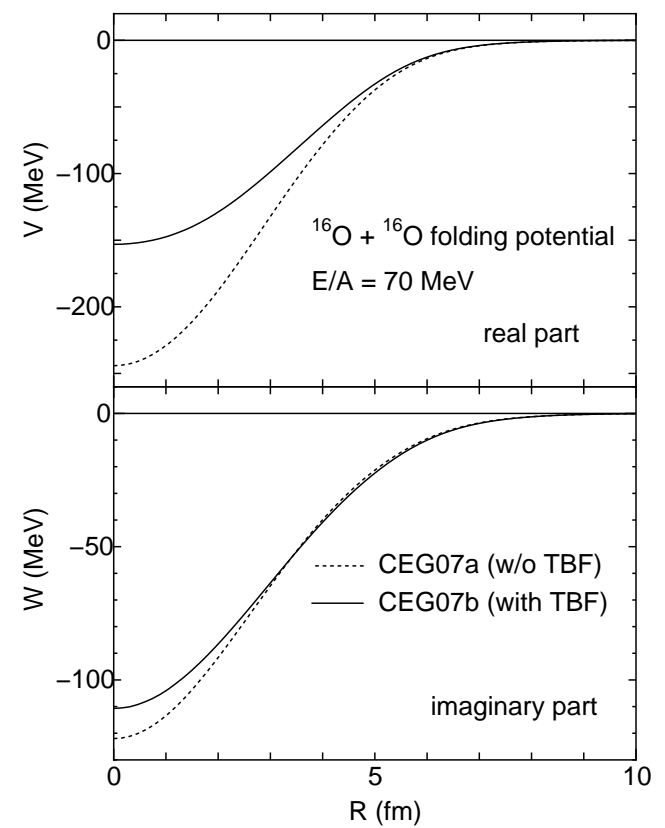

Fig. 2. The real and imaginary parts of the FMP for the ${ }^{16} \mathrm{O}+{ }^{16} \mathrm{O}$ system at $E / A=70 \mathrm{MeV}$. The dotted and solid curves are the results with CEG07a (without the TBF) and CEG07b (with the TBF), respectively.

to play only a minor role. The effects of the TBA and TBR will be shown later.

We then calculate the ${ }^{16} \mathrm{O}+{ }^{16} \mathrm{O}$ elastic-scattering cross section at $E / A=70 \mathrm{MeV}$ with the use of two types of FMPs shown in Fig. 2. In the standard DFM analyses of elastic scattering, it is often the case that the real part of calculated FMP is multiplied by the renormalization factor, whereas a completely phenomenological imaginary potential is introduced and the parameters together with the renormalization factor for the real FMP are determined so as to optimize the fit to the experimental data. In the present DFM, however, the calculated FMP itself is already complex because of the use of the complex $G$-matrix. The only parameter in the present framework is the renormalization factor $N_{\mathrm{W}}$ for the imaginary part defined by Eq. (14).

The results are shown in Fig. 3. The solid and dotted curves are the results with the use of the FMP obtained from CEG07b and CEG07a, respectively. Here, we take $N_{\mathrm{W}}$ to be 0.8 so that the solid curve (with the TBF effect) gives an optimum fit to the data. The solid curve with the TBF effect well reproduces the experimental data up to backward angles, while the dotted curve with CEG07a (without the TBF) overshoots the experimental data at middle and backward angles reflecting the too deep strength of the real part of the FMP. We found that no reasonable fit to the data was obtained by the FMP with CEG07a (without the TBF) no matter how the imaginary part of the FMP is renormalized by changing the value of $N_{\mathrm{W}}$ as seen in Fig. 4 . When we increase the $N_{\mathrm{W}}$ value so as to reduce the deviation in the large-angle region, no calculation reproduces the proper slope of the experimental cross sections at backward angles and the diffraction pattern in the

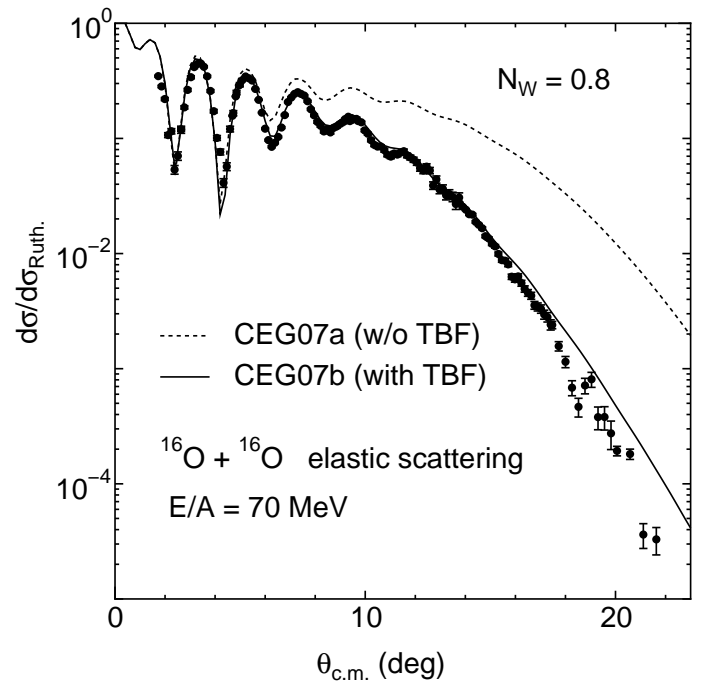

Fig. 3. Rutherford ratio of the cross sections for the ${ }^{16} \mathrm{O}+{ }^{16} \mathrm{O}$ elastic scattering at $E / A=70 \mathrm{MeV}$, which are compared with the folding model calculations with (CEG07b) and without (CEG07a) the TBF effect. The $N_{\mathrm{W}}$ value is fixed to 0.8 for all of the calculations. The meaning of the curves is the same as in Fig. 2. The experimental data is taken from Ref. [34].

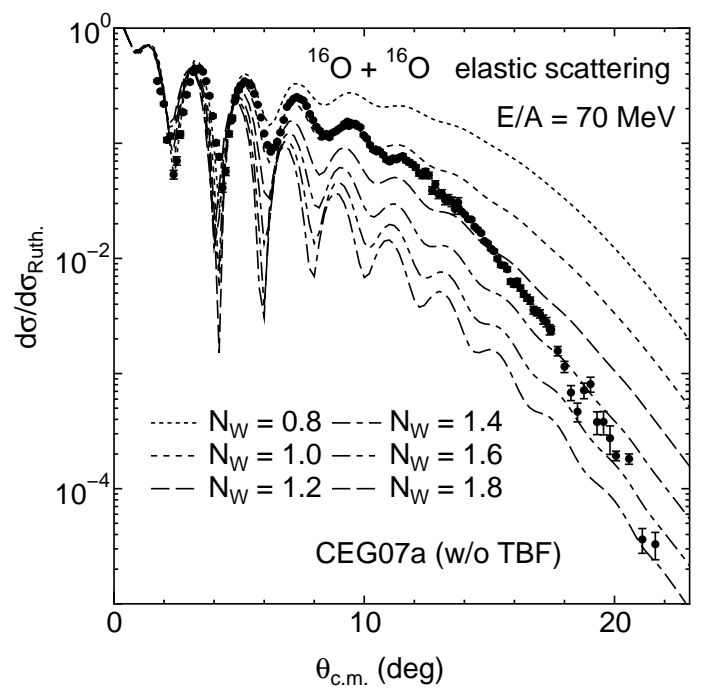

Fig. 4. Same as Fig. 3 but for the results of CEG07a with various renormalization factors for the imaginary part. This figure is taken from Ref. [8].

forward-angle region becomes out-of-phase with respect to the experimental data. Thus, the large difference between the solid and dotted curves in Fig. 3 clearly shows an evidence of the decisive role of the TBF on elastic scattering of the ${ }^{16} \mathrm{O}+{ }^{16} \mathrm{O}$ system.

Next, we analyze the individual effects of the TBA and TBR forces in ${ }^{16} \mathrm{O}+{ }^{16} \mathrm{O}$ elastic scattering. CEG07b includes the effect of the TBA in the form of the FujitaMiyazawa diagram and the one of the TBR simulated by the change of the vector meson masses. Here, we make 
clear whether the TBA or the TBR have important role in nucleus-nucleus elastic scattering.

First, we analyze the effect of the TBA. The TBA is known to be taken account of various diagrams, such as the $\Delta$ and Roper excitations. However, we assume that the TBA is due only to the Fujita-Miyazawa diagram with the $\Delta$ resonance because the $\Delta$ resonance is known to have the most important contribution to the TBA. On this assumption, we investigate the effect of the TBA strength on the nuclear matter properties as well as on the scattering cross section, by changing the TBA cut-off parameter. Here, we rename the three types of CEG07b with various TBA parameters as CEG07b0, CEG07b1 and CEG07b2 following Ref. [8]. CEG07b1 is the original CEG07b itself. CEG07b0 includes no thee-body attractive force. CEG07b2 has stronger TBA than CEG07b1, which is designed so that the most reasonable saturation curve is reproduced as shown in Fig. 5.

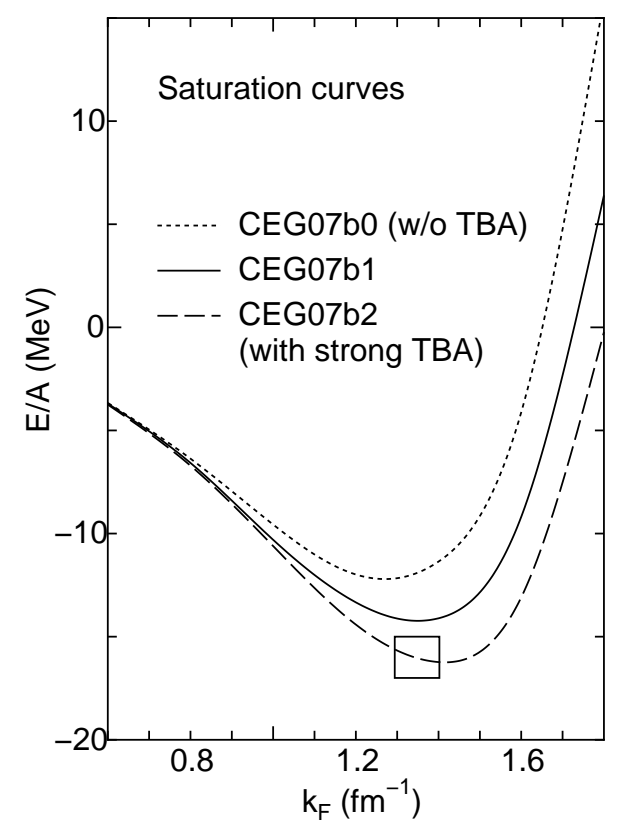

Fig. 5. Effects of the TBA in the saturation curves obtained by the $G$-matrix calculation. The dotted, solid and dashed curves are the results for CEG07b0, CEG07b1, and CEG07b2, respectively. This figure is taken from Ref. [8].

Figures 6 and 7 compare the cross sections calculated by the three types of CEG07b with experimental data in the use of the same and different $N_{\mathrm{W}}$ values, respectively. The solid, dotted and dashed curves are the results for CEG07b1, CEG07b0, and CEG07b2, respectively. While the effect of the TBA force is clearly seen in the saturation curves as shown in Fig. 5, this effect is seen little in the cross section as shown in Fig. 6. Moreover, this effect can be compensated by changing the renormalization factor $N_{\mathrm{W}}$ for the imaginary part by about $5 \%$ as shown in Fig. 7 and all of the calculated cross sections well reproduce the data. The role of the TBA effect is not clearly seen for nucleus-

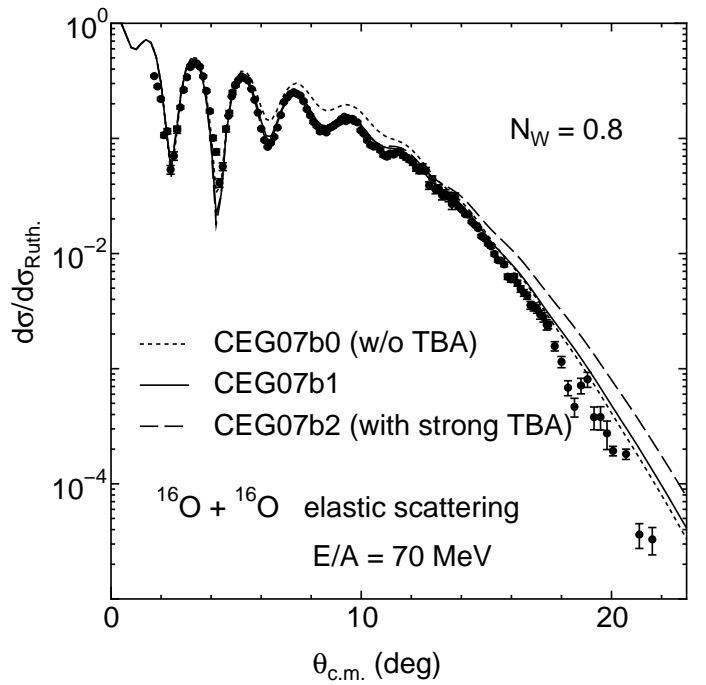

Fig. 6. Same as Fig. 3 but for the comparison with the effect of the three-body attractive force in the use of same $N_{\mathrm{W}}$ values. The meaning of the curves is the same as in Fig. 5.

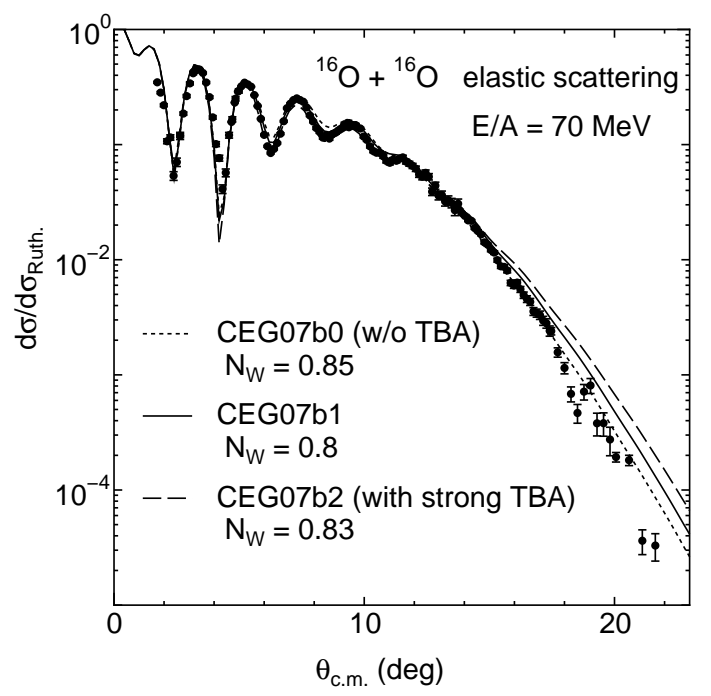

Fig. 7. Same as Fig. 6 but in the use of different $N_{\mathrm{W}}$ values. The meaning of the curves is the same as in Fig. 5. This figure is taken from Ref. [8].

nucleus elastic scattering although the $\Delta$ resonance (the Fujita-Miyazawa diagram) is known to be the most important TBA effect and clearly seen in the saturation curves in Fig. 5.

On the other hand, we also analyze the effect of the TBR force in nucleus-nucleus elastic scattering. The TBR effect of CEG07b is represented as the effective densitydependet two-body interaction by changing the vector meson masses in nuclear matter according to the functional form of $M_{V}(\rho)=M_{V} \exp \left(-\alpha_{V} \rho\right)$ with the parameter $\alpha_{V}$. To investigate the effect of the TBR strength on the nuclear matter properties as well as on the scattering cross section, we compare the CEG07b with different CEG07 fixed to 
be the parameter $\alpha_{V}=0.11$. Here, we name the CEG07 $\left(\alpha_{V}=0.11\right)$ as CEG07c2 in the same as Ref. [8].

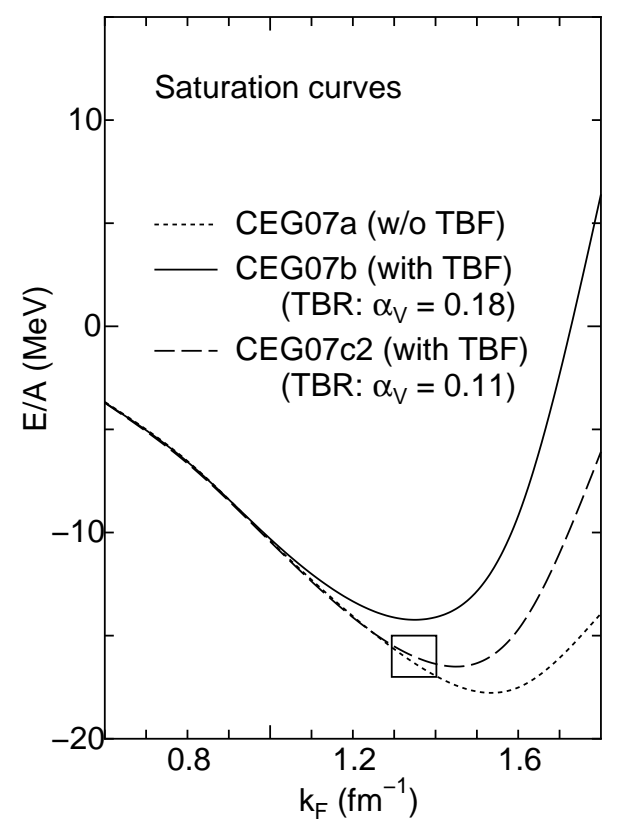

Fig. 8. Effect of the TBR in the saturation curves obtained by the $G$-matrix calculation. The dotted, solid and dashed curves are the results for CEG07a, CEG07b, and CEG07c2, respectively.

Figure 8 shows the saturation curves calculated with CEG07a, CEG07b and CEG07c2 in nuclear matter. The effect of the TBR is claerly seen in the higher densities and the effect becomes more and more remarkable as the density becomes higher. Figure 9 compare the cross sec-

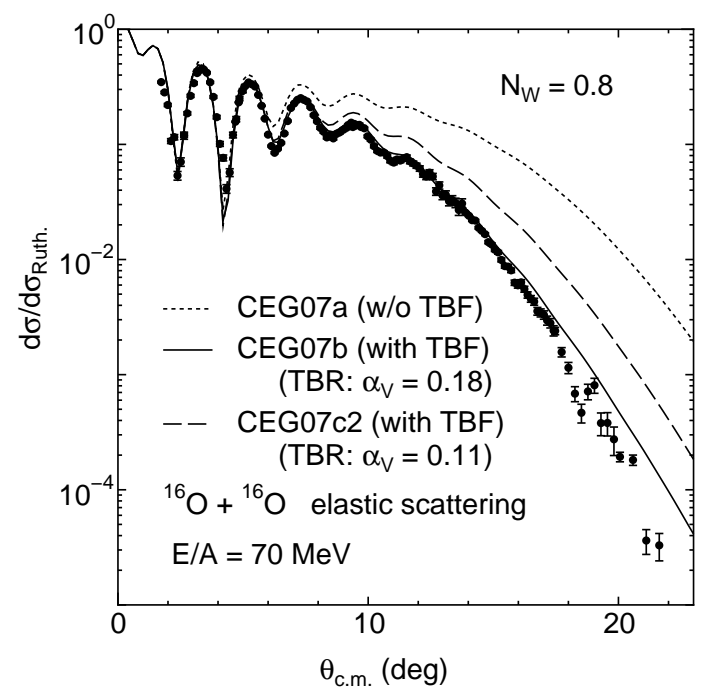

Fig. 9. Same as Fig. 6 but for the comparison with the effect of the three-body repulsive force. The meaning of the curves is the same as in Fig. 8. tions calculated by CEG07a, CEG07b and CEG07c2 with the experimental data. The solid, dotted and dashed curves are the results for CEG07b, CEG07a and CEG07c2, respectively. The effect of the TBR force is clearly seen in the cross section. Both CEG07a and CEG07c2 overshoot the data around middle and backward angles. Even if the imaginary part of CEG07c2 is renomalized by different $N_{\mathrm{W}}$ values, CEG07c2 cannot repuroduce the data in the same as CEG07a. This result means that the TBR effect has important role in nucleus-nucleus elastic scattering.

\subsection{Analysis of ${ }^{16} \mathrm{O}$ elastic scattering by other target nuclei}

In this section, we analyze the elastic scattering of ${ }^{16} \mathrm{O}$ by the ${ }^{12} \mathrm{C},{ }^{28} \mathrm{Si}$ and ${ }^{40} \mathrm{Ca}$ target nuclei at $E / A=93.9 \mathrm{MeV}$ with the use of CEG07a and CEG07b interactions.

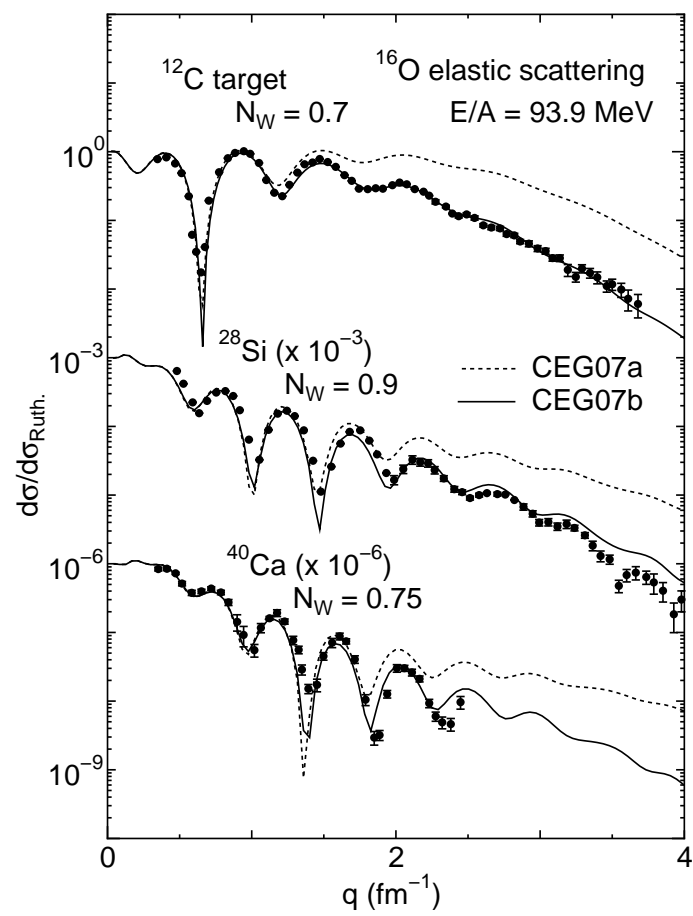

Fig. 10. Rutherford ratio of the cross sections for elastic scattering of ${ }^{16} \mathrm{O}$ by the ${ }^{12} \mathrm{C},{ }^{28} \mathrm{Si}$ and ${ }^{40} \mathrm{Ca}$ targets at $E / A=93.9$ $\mathrm{MeV}$, which are compared with the folding model calculations with the three types of complex $G$-matrix interactions. The dotted and solid curves are the results with CEG07a and CEG07b, respectively. The experimental data are taken from Ref. [36]. The abscissa is the momentum transfer $q$ defined as $q=2 k \sin \left(\frac{\theta}{2}\right)$, where $k$ being the asymptotic momentum.

Figure 10 shows the ${ }^{16} \mathrm{O}$ elastic-scattering cross sections for the ${ }^{12} \mathrm{C},{ }^{28} \mathrm{Si}$ and ${ }^{40} \mathrm{Ca}$ targets at $E / A=93.9 \mathrm{MeV}$. The dotted and solid curves are the results with CEG07a and CEG07b, respectively. The effect of the TBF is clearly seen in the cross sections as in the case of the ${ }^{16} \mathrm{O}+{ }^{16} \mathrm{O}$ scattering shown in Figs. 2. No reasonable fit to the data 
is obtained by the FMP calculation with CEG07a (without TBF effect), no matter how we change the value of $N_{\mathrm{W}}$, which is also the same as in the case of the ${ }^{16} \mathrm{O}$ target shown in Fig. 4. For the ${ }^{28} \mathrm{Si}$ target, the fit to the experimental data is not necessarily perfect at large angles. It may be related to the fact that the ${ }^{28} \mathrm{Si}$ nucleus presents a slightly stronger absorption $\left(N_{\mathrm{W}}=0.9\right)$ to the incident ${ }^{16} \mathrm{O}$ nucleus compared with other target nuclei $\left(N_{\mathrm{W}} \approx 0.75\right)$. Since the ${ }^{28} \mathrm{Si}$ nucleus is known to be a very deformed nucleus that shows a typical rotational band in the excitation spectrum, it may be reasonable to expect that an additional absorption should be induced dynamically by collective excitation of the ${ }^{28} \mathrm{Si}$ nucleus in the collision with ${ }^{16} \mathrm{O}$. This kind of dynamical effect may not be represented by the imaginary part of the $G$-matrix interaction evaluated in the nuclear matter. This would be one of the reason of a slightly larger value of the optimum $N_{\mathrm{W}}$ for the ${ }^{28} \mathrm{Si}$ target $\left(N_{\mathrm{W}}=\right.$ $0.9)$ compared with that for other targets $\left(N_{\mathrm{W}} \approx 0.75\right)$. In fact, we have confirmed that a better fit up to the backward angles is obtained by a coupled-channel (CC) calculation based on the present FMP potential in the case of the ${ }^{28} \mathrm{Si}$ target with a smaller value of $N_{\mathrm{W}}$. The CC analyses will be reported in forthcoming publications.

\section{$3.3{ }^{12} \mathbf{C}+{ }^{12} \mathrm{C}$ system}

In this section, we analyze elastic scattering of the ${ }^{12} \mathrm{C}+{ }^{12} \mathrm{C}$ system that has been studied at $E / A=135 \mathrm{MeV}$. The ${ }^{12} \mathrm{C}+{ }^{12} \mathrm{C}$ system is one of the interesting systems and the most frequently-studied light heavy-ion system.

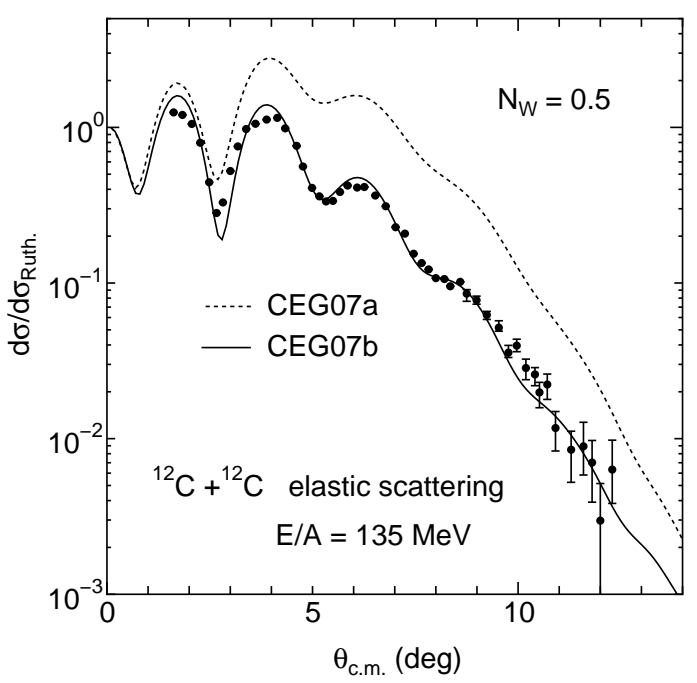

Fig. 11. Rutherford ratio for the ${ }^{12} \mathrm{C}+{ }^{12} \mathrm{C}$ system at $E / A=135$ $\mathrm{MeV}$. The experimental data are taken from Ref. [37].

Figures 11 shows the elastic-scattering cross sections for the ${ }^{12} \mathrm{C}+{ }^{12} \mathrm{C}$ system at $E / A=135 \mathrm{MeV}$, respectively. The dotted and solid curves are the results with CEG07a and CEG07b, respectively. The effect of the TBF is also clearly seen for the ${ }^{12} \mathrm{C}+{ }^{12} \mathrm{C}$ elastic scattering as in the case of other systems. This could be related to the weaker absorption (the optimum value of $N_{\mathrm{W}}=0.5$ ) for this system compared with other systems discussed so far, although the origin of the weaker absorption for this system is not clear at this stage. It is worthwhile to mention that the similar trend of the weaker absorption for elastic scattering relevant to the ${ }^{12} \mathrm{C}$ nucleus was also pointed out previously [38].

\section{Summary}

In summary, we have first analyzed elastic scattering of the ${ }^{16} \mathrm{O}+{ }^{16} \mathrm{O}$ system at $E / A=70 \mathrm{MeV}$ with the use of CEG07a and CEG07b interactions, where the latter one include the effect of the TBF (TBA + TBR). The effect of the TBF is seen clearly in the real potential, where the potential is pushed upward remarkably by the TBR contribution in the high-density region owing to the prescription of the frozen-density approximation (FDA). Then, the DFM with CEG07b including the TBR effect nicely reproduces the observed ${ }^{16} \mathrm{O}+{ }^{16} \mathrm{O}$ elastic-scattering cross sections over the whole angular region. On the other hand, the DFM with CEG07a derived only from the two-body interaction gives no reasonable fit to the data no matter how the value of $N_{\mathrm{W}}$ is changed.

The effect of the $\Delta$-resonance TBA that is described by the Fujita-Miyazawa diagram have been tested. The TBA effect is clearly seen in the saturation curves and has an important role to satisfy the saturation point. However, the effect can be compensated by changing the renormalization factor $N_{\mathrm{W}}$ for the imaginary part by about $5 \%$ for the ${ }^{16} \mathrm{O}+{ }^{16} \mathrm{O}$ elastic scattering at $E / A=70 \mathrm{MeV}$. It is understood that the TBR-like effect of the TBF has a decisive role in nucleus-nucleus elastic scattering. The effect of the TBR have also been tested. Differently from the TBA effect, the TBR effect is clearly seen in the cross section. The effect of TBR force has desicive role to repuroduce the nucleus-nucleus elastic scattering data.

We also apply the folding model potential calculated with CEG07a and CEG07b in the framework of the FDA for other systems; the ${ }^{16} \mathrm{O}+{ }^{12} \mathrm{C},{ }^{16} \mathrm{O}+{ }^{28} \mathrm{Si}$, and ${ }^{16} \mathrm{O}+{ }^{40} \mathrm{Ca}$ systems at $E / A=93.9 \mathrm{MeV}$ and the ${ }^{12} \mathrm{C}+{ }^{12} \mathrm{C}$ system at $E / A=135 \mathrm{MeV}$. All the cross sections can be reproduced by the calculated FMPs with CEG07b and CEG07c (with the TBF). The FMP calculated with CEG07a (without the TBF) can not reproduce the data as in the case of the ${ }^{16} \mathrm{O}+{ }^{16} \mathrm{O}$ system. CEG07a gives too deep FMP to reproduce the data. The effect of the TBF is also clearly seen at FMP and elastic cross sections for these systems as in the case of the ${ }^{16} \mathrm{O}+{ }^{16} \mathrm{O}$ at $E / A=70 \mathrm{MeV}$.

\section{Acknowledgments}

The authors acknowledge Professor Dao T. Khoa for providing with the numeral data of the ${ }^{16} \mathrm{O}+{ }^{16} \mathrm{O}$ elastic scattering. One of the authors (T.F.) is supported by the Japan Society for the Promotion of Science for Young Scientists. 
$19^{\text {th }}$ International IUPAP Conference on Few-Body Problems in Physics

\section{References}

1. F. Coester, S. Cohen, B. D. Day and C. M. Vincent, Phys. Rev. C 1 (1970) 769.

2. I. E. Lagaris and V. R. Pandharipande, Nucl. Phys. A359 (1981) 349.

3. R. B. Wiringa, V. Fiks and A. Fabrocini, Phys. Rev. C 38 (1988) 1010.

4. M. Baldo, I. Bombaci and G. F. Burgio, Astron. Astrophys. 328 (1997) 274.

5. A. Lejeune, U. Lombardo and W. Zuo, Phys. Lett. B477 (2000) 45.

6. T. Furumoto, Y. Sakuragi and Y. Yamamoto, Phys. Rev. C 79 (2009) 011601.

7. T. Furumoto, Y. Sakuragi and Y. Yamamoto, Phys. Rev. C 78 (2008) 044610.

8. T. Furumoto, Y. Sakuragi and Y. Yamamoto, Phys. Rev. C 80 (2009) 044614.

9. T. A. Rijken, Phys. Rev. C 73 (2006) 044007.

10. T. A. Rijken and Y. Yamamoto, Phys. Rev. C 73 (2006) 044008.

11. J. Fujita and H. Miyazawa, Prog. Theor. Phys. 17 (1957) 360.

12. D. T. Khoa, W. von Oertzen and H. G. Bohlen, Phys. Rev. C 49 (1994) 1652.

13. D. T. Khoa, G. R. Satchler and W. von Oertzen, Phys. Rev. C 56 (1997) 954.

14. G. Bertsch, J. Borysowicz, H. McManaus and W. G. Love, Nucl. Phys. A284 (1977) 399.

15. T. Kasahara, Y. Akaishi and H. Tanaka, Prog. Theor. Phys. Suppl. 56 (1974) 96.

16. B. Sinha, Phys. Rep. 20 (1975) 1.

17. B. Sinha and S. A. Moszkowski, Phys. Lett. B81 (1979) 289.

18. J. W. Negele and D. M. Vautherin, Phys. Rev. C 5 (1972) 1472

19. X. Campi and A. Bouyssy, Phys. Lett. B73 (1978) 263.

20. D. T. Khoa, Phys. Rev. C 63 (2001) 034007.

21. S. Nagata, M. Kamimura and N. Yamaguchi, Prog. Theor. Phys. 73 (1985) 512.

22. M. Katsuma, Y. Sakuragi, S. Okabe and Y. Kondo, Prog. Theor. Phys. 107 (2002) 377.

23. G. R. Satchler and W. G. Love, Phys. Rep. 55 (1979) 183.

24. F. Duggan, M. Lassaut, F. Michel and N. V. Mau, Nucl. Phys. A355 (1981) 141.

25. F. Carstoiu and M. Lassau, Nucl. Phys. A597 (1996) 269.

26. T. Furumoto and Y. Sakuragi, Phys. Rev. C 74 (2006) 034606.

27. S. Okabe, Proc. of Tours Symposium on Nuclear Physics II (eds. H. Utsunomiya et al., World Scientific, Singapore, 1995), p. 112.

28. H. DeVries, C. W. DeJager and C. DeVries, At. Data Nucl. Data Tables 36 (1987) 495.

29. L. R. B. Elton, Nuclear Size (Oxford University, Oxford, 1961).

30. Y. Kondō, F. Michel, and G. Reidemeister, Phys. Lett. B242, 340 (1990).
31. Y. Kondō, Y. Sugiyama, Y. Tomita, Y. Yamanouchi, H. Ikezoe, K. Ideno, S. Hamada, T. Sugimitsu, M. Hijiya and H. Fujita, Phys. Lett. B365 (1996) 17.

32. M. E. Brandan and G. R. Satchler, Phys. Rep. 285 (1997) 143, and references therein.

33. N. Yamaguchi, S. Nagata and T. Matsuda, Prog. Theor. Phys. 70 (1983) 459.

34. F. Nuoffer, G. Bartnitzky, H. Clement, A. Blazevic, H. G. Bohlen, B. Gebauer, W. von Oertzen, M. Wilpert, Th. Wilpert, A. Lepine-Szily, W. Mitting, A. N. Ostrowski and P. Roussel-Chomaz, Nuovo Cimento A 111 (1998) 971.

35. D. T. Khoa, W. von Oertzen, H. G. Bohlen and S. Ohkubo, J. Phys. G: Nucl. Part. Phys. 34 (2007) R111.

36. P. Roussel-Chomaz, N. Alamanos, F. Auger, J. Barrette, B. Berthier, B. Fernandez and L. Papineau, Nucl. Phys. A477 (1988) 345.

37. T. Ichimura, T. Niizeki, H. Okamura, H. Ohnuma, H. Sakai, Y. Fuchi, K. Hatanaka, M. Hosaka, S. Ishida, K. Kato, S. Kato, H. Kawashima, S. Kubono, S. Miyamoto, H. Orihara, N. Sakamoto, S. Takaku, Y. Tajima, M. H. Tanaka, H. Toyokawa, T. Uesaka, T. Yamamoto, T. Yamashita, M. Yosoi and M. Ishihara, Nucl. Phys. A569 (1994) 287.

38. K. Schwarz, C. Samanta, M. Fujiwara, H. Rebel, R. De Leo, N. Matsuoka, H. Utsunomiya, H. Akimune, I. Daito, H. Fujimura, F. Ihara, K. Ishibashi, Y. Maeda, T. Yamanaka, H. Yoshida, A. Okihana, T. Yoshimura, P.K.J. van Aarle, W.A.T. Uijen, M. Ito and Y. Sakuragi, Eur. Phys. J. A 7 (2000) 367. 